\title{
Identification of an infectious progenitor for the multiple-copy HERV-K human endogenous retroelements
}

\author{
Marie Dewannieux, ${ }^{1,3}$ Francis Harper, ${ }^{2,4}$ Aurélien Richaud, ${ }^{1,4}$ Claire Letzelter, ${ }^{1}$ \\ David Ribet, ${ }^{1}$ Gérard Pierron, ${ }^{2}$ and Thierry Heidmann ${ }^{1,5}$ \\ ${ }^{1}$ Unité des Rétrovirus Endogènes et Eléments Rétroïdes des Eucaryotes Supérieurs, UMR 8122 CNRS, Institut Gustave Roussy, \\ 94805 Villejuif Cedex, France; ${ }^{2}$ Laboratoire de Réplication de I'ADN et Ultrastructure du Noyau, UPR1983 Institut André Lwoff, \\ 94801 Villejuif Cedex, France
}

\begin{abstract}
Human Endogenous Retroviruses are expected to be the remnants of ancestral infections of primates by active retroviruses that have thereafter been transmitted in a Mendelian fashion. Here, we derived in silico the sequence of the putative ancestral "progenitor" element of one of the most recently amplified family-the HERV-K family-and constructed it. This element, Phoenix, produces viral particles that disclose all of the structural and functional properties of a bona-fide retrovirus, can infect mammalian, including human, cells, and integrate with the exact signature of the presently found endogenous HERV-K progeny. We also show that this element amplifies via an extracellular pathway involving reinfection, at variance with the non-LTR-retrotransposons (LINEs, SINEs) or LTR-retrotransposons, thus recapitulating ex vivo the molecular events responsible for its dissemination in the host genomes. We also show that in vitro recombinations among present-day human $H E R V-K$ (also known as ERVK) loci can similarly generate functional HERV-K elements, indicating that human cells still have the potential to produce infectious retroviruses.
\end{abstract}

[Supplemental material is available online at www.genome.org.]

Nearly $8 \%$ of the human genome is composed of sequences of retroviral origin. Most of them are degenerate, either due to recombination between the two provirus LTRs or to mutations interrupting the retroviral ORFs. The env gene seems to be best conserved, with 18 intact genes retaining a full coding capacity in the human genome (Benit et al. 2001; de Parseval et al. 2003; Villesen et al. 2004), possibly because of its potential role in human physiology. The HERV-K(HML2) family of endogenous retroviruses is an exception to this general rule, since some copies still contain complete ORFs for the other retroviral genes (Löwer et al. 1996; for review, see Bannert and Kurth 2004). This family includes the most recently amplified endogenous retroviruses, most of which have integrated into the genome $<5$ million years ago, with a few insertions showing polymorphism within the human population (Steinhuber et al. 1995; Medstrand and Mager 1998; Barbulescu et al. 1999; Turner et al. 2001; Hughes and Coffin 2004; Belshaw et al. 2005). Some of these recently integrated proviruses are responsible for the synthesis of retroviral particles that can be observed in teratocarcinoma and melanoma-derived cell lines (Boller et al. 1993; Löwer et al. 1993; Bieda et al. 2001; Muster et al. 2003; Buscher et al. 2005), and possibly in human placenta (Kalter et al. 1973; Dirksen and Levy 1977; Wilkinson et al. 1994). Because of this "activity," the HERV-K(HML2) family has been the subject of numerous studies in the past years, with the description of alleles with nearly intact

${ }^{3}$ Present address: Department of Immunology and Molecular Pathology, University College of London, Windeyer Institute, London W1T 4JF, UK.

${ }^{4}$ These authors contributed equally to this work.

${ }^{5}$ Corresponding author.

E-mail heidmann@igr.fr; fax 33-1-42-11-53-42.

Article published online before print. Article and publication date are at http:// www.genome.org/cgi/doi/10.1101/gr.5565706. proviruses and complete coding capacity (Mayer et al. 1999; Reus et al. 2001; Turner et al. 2001). Despite these efforts, no functional provirus able to produce infectious particles has yet been described. Even if several loci containing complete ORFs have been identified, and in spite of the availability of the complete sequence of the human genome, the search for a functional provirus is hampered by the significant polymorphism that can be detected at each HERV-K(HML2) locus within the human population. To overcome this limitation, we used a reverse strategy and generated a consensus HERV-K(HML2) provirus, thus "resuscitating" Phoenix, the likely progenitor of the last, humanspecific HERV-K(HML2) amplification burst. This allowed a definite characterization of the structure and life cycle of an "ancestral" retroviral element and recapitulation of the molecular events responsible for its amplification.

\section{Results}

Phoenix, the ancestral HERV-K(HML2) retrovirus

To construct a consensus HERV-K(HML2) provirus, we assembled all of the complete copies of the $9.4-\mathrm{kb}$ proviruses that are human specific (excluding those with the 292-nt deletion at the beginning of the env gene) and aligned their nucleotide sequence to generate the consensus in silico, taking for each position the most frequent nucleotide. The resulting provirus sequence contains, as expected, ORFs for all of the HERV-K(HML2)-encoded proteins (Gag, Pro, Pol, Env, and the accessory Rec protein), with gag, pro, and pol separated by -1 frameshifts. Noteworthily, this consensus provirus is distinct from each of the sequences used to generate it, with at least 20 amino acid changes on the overall sequences (Fig. 1).

Based on this in silico reconstruction, we then generated a 
A

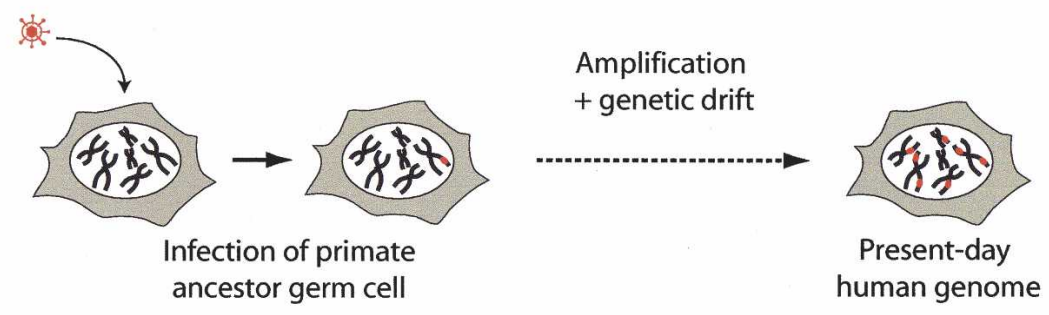

B

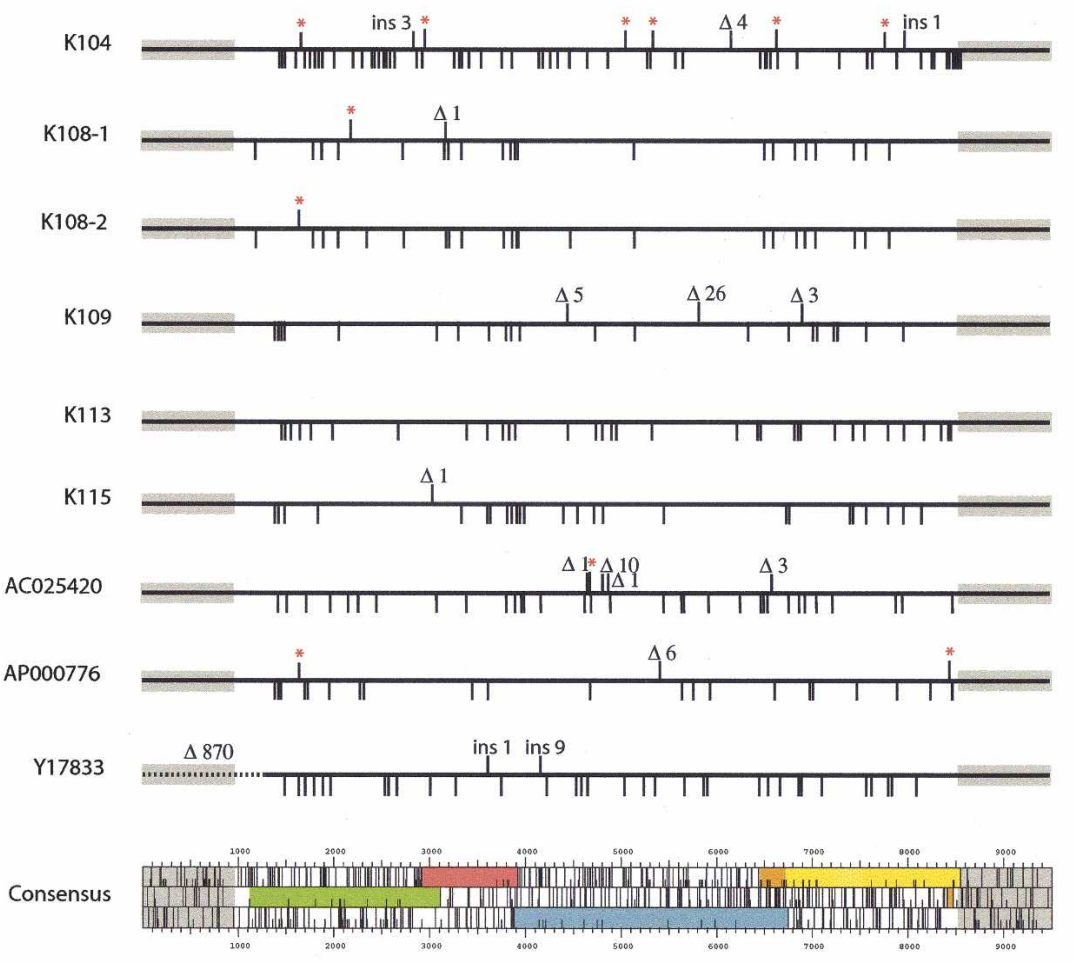

RNA

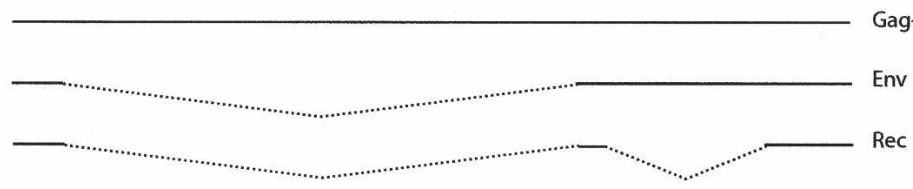

Figure 1. HERV-K(HML2) "endogenization" and present-day human proviruses. (A) Evolutionary scheme for HERV-K(HML2) entry into and invasion of the genome of primates. (B) Map of the fulllength 9.4-kb long human-specific HERV-K(HML2) proviruses and comparison with the in silicoengineered consensus sequence. Each provirus is represented by a solid dark line, with the amino acid substitutions in Gag, Pro, Pol, and Env as compared with the consensus element indicated below the line, and the insertions/deletions (ins/ $\Delta$ ) and premature Stop codons (red stars) indicated above the line. The ORF map of the consensus provirus is shown, with gag in green, pro in pink, pol in blue, env in orange and yellow, the bipartite rec in orange, and the two LTRs as gray boxes. (Note that the first coding exon of rec belongs to the env ORF). The transcripts responsible for the expression of the viral proteins, with the corresponding spliced out domains (dotted lines), are schematized below the ORF map.

molecular clone corresponding to the consensus DNA proviral sequence that we named Phoenix, using the related K108 and K109 proviruses (which we had previously cloned from a commercial human BAC library) as a backbone, and a commercial kit to introduce single nucleotide mutations at each position required to match the consensus. As the HERV-K(HML2) LTRs are not functional in every cell line (Ruda et al. 2004; Lavie et al. 2005), we also replaced the U3 part of the 5' LTR by the CMV promoter, with its start site positioned so as to conserve the expected nucleotide sequence of the native retroviral transcript. We also introduced a cloning site downstream of the env gene into the noncoding U3 region of the 3' LTR to possibly tag (with the neo gene) the retroviral transcript without altering its coding capacity.

\section{Phoenix codes for bona-fide retroviral particles}

In a first assay, we introduced the Phoenix expression vector into human 293T cells, and looked for retroviral particles by transmission electron microscopy. As illustrated in Figure 2, A and B, this resulted in the synthesis of particles budding from the plasma membrane (not observed with a control vector), as classically observed with $\gamma$ - (type-C) or $\delta$ (HTLV) retroviruses, or even lentiviruses (HIV). No preassembled particles were detected within the cytoplasm, although HERV-K(HML2) elements are more closely related to $\beta$ - (type-B/D) retroviruses based on their pol gene (but this is consistent with viral particle morphology and site of assembly being essentially dependent on the gag rather than the pol gene). In the extracellular space, two classes of particles could be observed, either with a hollow interior surrounded by a dense ring of stain corresponding to immature particles or with a condensed hexagonal core corresponding most probably to the mature form of the virus (Fig. 2C), in which the polyproteins are cleaved into the final products. Consistently, a provirus with a mutation introduced into the active site of the protease (Pro) also produces particles, but all of them disclose the immature morphology (Fig. 2F). The particles are surrounded by prominent spikes (Fig. 2D) that can be marked in immunoelectron microscopy experiments with an antibody directed against the SU subunit of the HERV-K(HML2) envelope protein (Fig. 2E). As expected, these spikes are absent when the provirus contains a premature Stop codon in the env gene (Fig. 2G). Altogether, these features suggest that Phoenix can direct the synthesis of complete retroviral particles.

As a confirmation, we performed immunoblot analyses of the 293T cells transfected with the proviral vector. As illustrated in Figure 3A, Phoenix, as well as its pro mutant, promotes the synthesis of a Gag precursor of $\sim 80 \mathrm{kDa}$ in cell lysates, as well as some cleaved products (not observed with the pro mutant). Cleavage products could also be observed in the supernatant of 

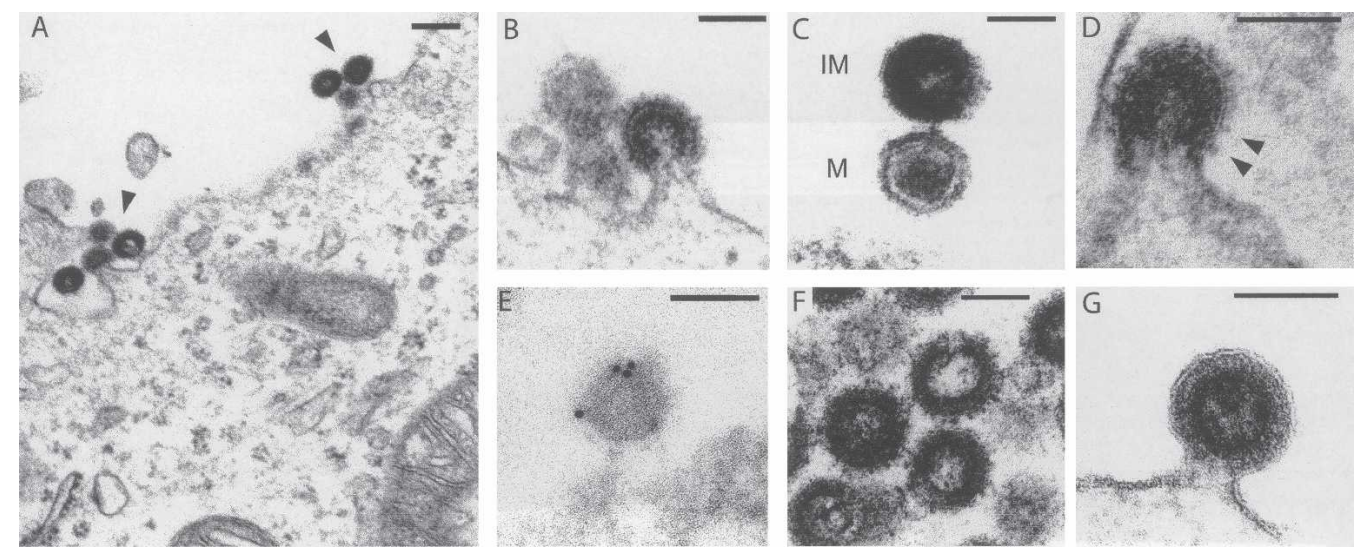

Figure 2. Electron microscopy of the viral-like particles generated by the Phoenix provirus. Human 293T cells were transfected with an expression vector for Phoenix $(A-E)$, or mutants $(F, G)$, and observed $48 \mathrm{~h}$ post-transfection. $(A)$ Low magnification of particles assembled at the cell membrane. (B) Representative image of particles budding from the plasma membrane. (C) High magnification of two particles, one of which (bottom) discloses a mature (M) morphology with a condensed core, while the other appears to be still immature (IM) with two dark peripheral rings surrounding an electron-lucent core. $(D)$ High magnification of a particle with prominent spikes, corresponding to the Env protein. (E) Image of a particle after labeling with an antibody specific for the HERV-K envelope protein and a secondary antibody linked to gold beads, obtained by immuno-electron microscopy. Quantification of the labeling on 11 independent fields demonstrates association of the gold beads with the viral particles: $307 \pm 121$ gold beads/ $\mu \mathrm{m}^{2}$ for the viral particles, versus $4.9 \pm 3.2$ and $1.1 \pm 1.5$ gold beads $/ \mu \mathrm{m}^{2}$ for the cytoplasm and particle-free extracellular space, respectively $(P<0.001$ between viral particles and any of the two other compartments, Student's $t$-test). ( $F$ ) Image of representative particles obtained after transfection with an expression vector for the Phoenix pro mutant. All of them disclosed an immature morphology (41 of 41 identified "free" particles, i.e., no more in the budding process, for the pro mutant, vs. 15 of 37 for Phoenix WT). (G) High magnification of a particle obtained after transfection with an expression vector for the Phoenix env mutant. The membrane surrounding the particle is clearly detectable, without any spike. Scale bars: $(A): 200 \mathrm{~nm},(B-G): 100 \mathrm{~nm}$.

the transfected cells, except for the pro mutant that only expresses the Gag precursor. Similarly, using an antibody directed against the SU component of the HERV-K(HML2) envelope protein, we detected, in the supernatants, a protein of a size $(55 \mathrm{kDa})$ consistent with that of the processed SU subunit, but we were unable to detect the Env protein in cell lysates, most probably because of a too-low expression level. Notwithstanding, expression of the envelope protein could be observed by immunofluorescence analysis using HeLa and 293T cells transiently transfected with Phoenix (Fig. 3B). In these cells, it can further be observed that Gag and Env colocalize to some common subcellular domains including, as expected, the plasma membrane.

\section{Phoenix is an infectious retrovirus}

To get insight into the functionality of this ancestral virus, we then looked for a reverse-transcriptase activity in the supernatant of the transfected cells. Using the previously described sensitive product-enhanced reverse transcriptase (PERT) assay (Pyra et al. 1994; see Methods), an RT activity could indeed be detected in the supernatant of cells transfected with Phoenix, not observed with a provirus in which we had introduced a mutation within the catalytic site of the RT domain (Fig. 3C). To further study the infectivity potential of Phoenix-derived particles, we then infected a panel of mammalian cell lines with the supernatant of 293T cells transfected with a neo-marked Phoenix provirus, and subjected them to G418 selection. G418 ${ }^{\mathrm{R}}$ clones were obtained for several target cells including hamster BHK21 cells, feline G355.5 cells, and, noteworthily, the human SH-SY5Y and 293 cells, indicating that Phoenix is fully functional and infectious (Fig. 4A). In the same assay, the human HeLa cells and the murine WOP cells seemed to be resistant to infection, most probably due to the absence of the appropriate receptor for the Env protein at the cell surface. We then ensured that the $G 418^{\mathrm{R}}$ clones that were obtained are the result of bona-fide infection. Their occur- rence actually depends on the presence of intact gag, pro, pol, and env genes, since the inactivation of any of the encoded proteins renders Phoenix noninfectious without decreasing the amount of viral particles produced, except for the gag mutant, as assessed by Western Blot (Fig. 4A; data not shown). In these experiments, the apparent titer of Phoenix is quite low even for the cell lines permissive for infection. A quantitative assay by real-time PCR on the genomic DNA from BHK21 cells infected with particles generated by Phoenix, marked, or not, by the neo gene (see Methods), indicated that the presence of neo within the provirus contributes only to a limited extent to this low titer. A possible explanation is that infection with Phoenix occurs more efficiently via cell-cell interaction, as observed for HTLV and in some cases HIV (see Discussion), consistent with some images obtained by electron microscopy where budding particles appear to be directly captured by a recipient cell (Fig. 4B).

Next, we characterized further the $G 418^{\mathrm{R}}$ clones that we isolated and determined the provirus insertion sites using an inverse PCR strategy. Identification of the target loci of these new insertions was further asserted by direct PCR reactions between the identified flanking sequences and the neo gene of the marked proviruses (see Methods). In all cases, we found complete LTRs with short target-site duplications (TSDs) bordering the newly inserted proviruses (Fig. 5), thus confirming that the infection process is canonical. Rather surprisingly, the TSDs were of a variable length (one of $5 \mathrm{bp}$ and two of $6 \mathrm{bp}$ ), which was unexpected since well-characterized retroviral integrases yield TSDs of a fixed length (for review, see Brown 1997). However, our data are consistent with the structures observed for the HERV-K(HML2) endogenous copies of the human genome, since, even if the most common length of the endogenous HERV-K proviruses TSD is 6 bp, we could identify, both in the literature (Barbulescu et al. 1999; Turner et al. 2001) and databases (our unpublished observation), a few elements (three of 15 full-length proviruses) bordered by perfect TSD of only 5 bp (see the endogenous K115

\section{Genome Research}

www.genome.org 


\section{A functional HERV-K human endogenous retrovirus}

A

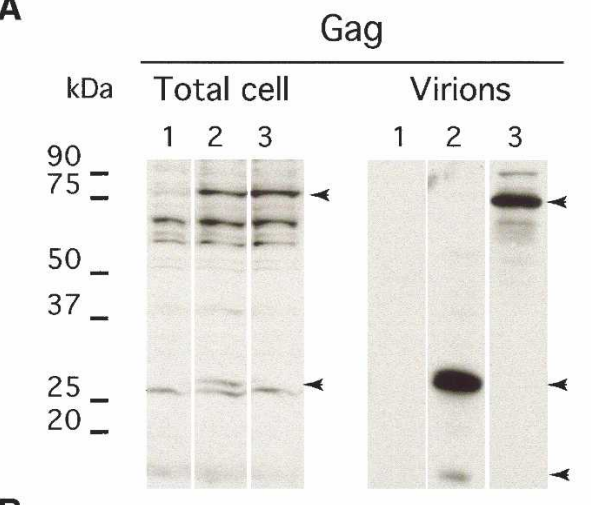

B
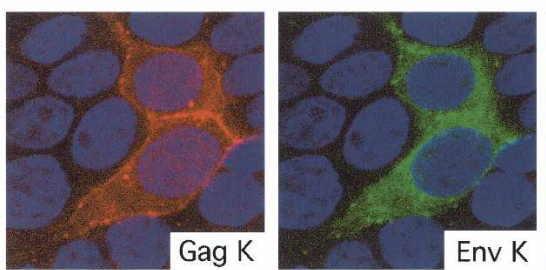

C

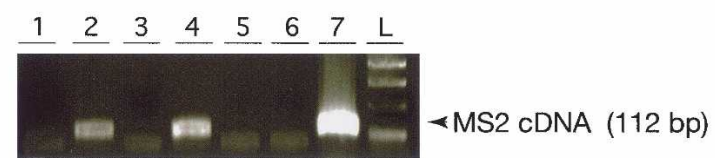

Figure 3. Characterization of Phoenix. $(A)$ Immunoblot analysis of the Phoenix products present in the lysates of transiently transfected 293T cells, or in the concentrated supernatant. Cells were transfected with (1) a control plasmid (pCMV- $\beta$ ), (2) an expression vector for Phoenix, or (3) an expression vector for a pro mutant. The membranes were hybridized with an antibody directed against the Gag protein (left) or the Env protein (right). (B) Immunofluorescence study of 293T cells transfected with Phoenix. Gag is marked in red, Env in green, and the nuclei are stained in blue. Note the colocalization of the two proteins, especially at the membrane that separates the two adjacent marked cells. (C) Detection of RT activity in the concentrated supernatant of 293T cells transfected with Phoenix and a series of proviral and control vectors. Concentrated virions were used as a source of RT activity to reverse transcribe synthetic MS2 RNA. Presence of cDNA was revealed via a PCR amplification using appropriate primers. (1) pCMV- $\beta$; (2) Phoenix; (3) Phoenix mutated in RT; (4) chimera K109-K115; (5) chimera K109-K113; (6) chimera K109-K108; (7) MLV core proteins).

provirus in the lower part of Fig. 5 for an illustration). Finally, we mapped the three Phoenix insertion sites using the UCSC Genome Browser and found that integration occurred within (for two of three) or close to $(<20 \mathrm{~kb}$, for one of three) genes. Although the number of target sites that we characterized is low, and thus cannot reveal subtle insertion site preferences, the identified loci are consistent with the preferences observed for classical retroviruses that favor insertion in transcribed regions (for review, see Bushman 2003). Interestingly, the characterization of the insertion sites of 11 (nearly) full-length HERV-K(HML2) endogenous elements gave a rather different result, with five of them found far from known human genes $(>100 \mathrm{~kb}$ on each side), four being in the vicinity $(<20 \mathrm{~kb})$ of genes, and only two inserted within genes (M. Dewannieux, unpubl.). This discrepancy is probably due to the strong counter-selection that should operate in vivo against insertions within genes, which are most probably deleterious for the proper expression of the targeted genes.

\section{HERV-K(HML2) provirus amplification via reinfection}

Next, to get an insight into the possible physiological mechanism responsible for the amplification of the HERV-K(HML2) family of endogenous retroviruses, we analyzed the relative efficiency of intracellular retrotransposition versus reinfection by Phoenix. To do so, we marked Phoenix with a specific neo indicator gene $\left(n e o^{\mathrm{TNF}}\right)$, in which neo becomes active only after the marked element has been transcribed, reverse-transcribed, and integrated (Esnault et al. 2002). The marked element was introduced by transfection into various cell lines, permissive or not, for infec-

A
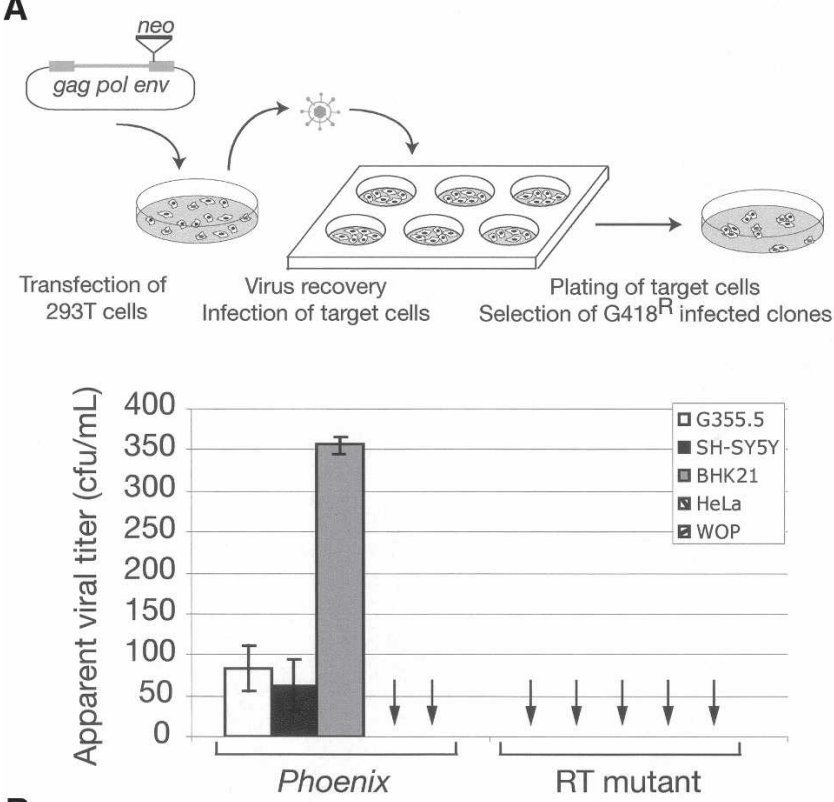

B

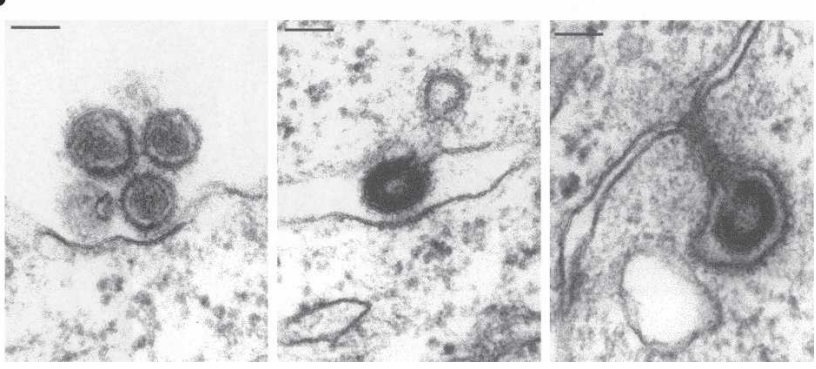

Figure 4. Infectious properties of Phoenix encoded particles. (A) Infectivity of the HERV-K retroviral particles. The apparent viral titer observed with the neo-marked HERV-K(HML2) provirus is indicated for a panel of target cell lines (the quantitative results for the 293 cell line is not shown since their restricted adherence impaired the proper quantification of individual $\mathrm{G} 418^{\mathrm{R}}$ clones), together with that obtained with a mutant for the RT domain tested in the same conditions (no clone observed); data are the results from three to eight independent experiments, with the standard deviation indicated. The other mutants (gag, pro, and env) gave the same, negative, results when assayed, with no clone observed in three independent experiments (difference measured between Phoenix WT and mutants significant with $P<0.001$ for G355.5 and BHK21 cells and $P$ $<0.01$ for SH-SY5Y cells, Student's $t$-test). The rationale of the assay is schematized at top. (B) Infection of cells by Phoenix particles. On the left, two mature particles appear to interact with the cell membrane of the closest cell, with a thickening of the membrane at the exact point of interaction with the particle. In the middle and on the right, two images suggestive of a cell-to-cell infection are presented, with a particle still budding from its progenitor cell already in contact with the neighboring cell. 


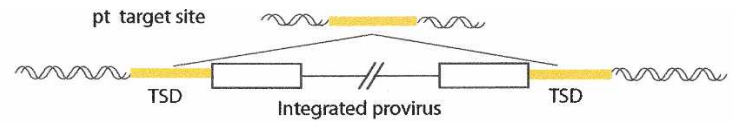

De novo Phoenix provirus insertions

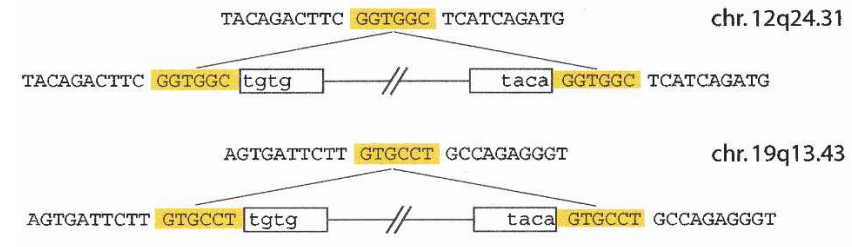

TTTGATAGTTA CTATG tgtg chr

\section{Natural HERV-K provirus insertion polymorphisms}

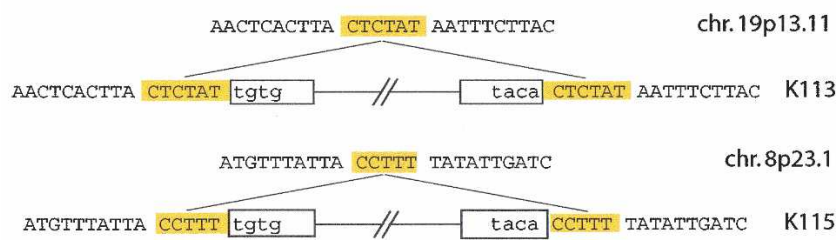

Figure 5. Structure of three de novo integration sites of Phoenix provirus, and comparison with the structure of natural HERV-K provirus insertions. The complete characterization of inserted Phoenix elements and insertion sites was performed using individual clones from human $\mathrm{SH}$ SY5Y cells after infection and G418 selection. A provirus insertion and the corresponding empty site are schematized at the top. The sequences of three characterized Phoenix de novo insertions are shown below, with the flanking DNA in uppercase and the proviral sequences in lowercase; target-site duplications of $5 / 6$ bp (TSD, yellow) are found in all cases, associated with full-length LTRs. For comparison, the corresponding structures of two resident HERV-K proviruses with a polymorphic insertion in humans (Turner et al. 2001) are presented in the lower part, one with a 5-bp TSD and one with a 6-bp TSD.

tion. Under these conditions, we reproductively obtained G418 ${ }^{\mathrm{R}}$ clones only with the cell lines permissive for infection (i.e., the feline G355.5 cells, the hamster BHK21 cells, and the human SH-SY5Y cells) (Fig. 6; data not shown). This suggests that the amplification process must, at some stage, get the envelope protein receptor involved, and thus should occur via an extracellular reinfection process. To confirm this hypothesis, we performed the same experiment as above, but with the provirus defective for the env gene (see Methods), using SH-SY5Y permissive cells. Under these conditions, no amplification of the marked mutant provirus was observed. As a control, addition of a CMV-driven expression vector for the envelope protein restored, at least partially, the mobility of the env-defective provirus (Fig. 6) (partial restoration might be due, indirectly, to an identified cytotoxic effect of Env overexpression, as commonly observed with this class of retroviral transmembrane proteins). Altogether, the results strongly support the hypothesis that HERV-K(HML2) amplification in the human genome took place via a reinfection process, possibly in the germ line of the developing embryo, a process consistent both with evolutionary data indicating that the HERV-K(HML2) env gene has been subjected to the same purifying selection as the other retroviral genes during amplification of this family (Belshaw et al. 2004) and with the presence of retroviral particles in human placenta and in germ-line tumors morphologically similar to those produced by Phoenix (Kalter et al. 1973; Dirksen and Levy 1977; Boller et al. 1993; Löwer et al. 1993; Wilkinson et al. 1994).

\section{“Recombination" of human endogenous HERV-K(HML2) loci can generate an infectious retrovirus}

Finally, taking advantage of our present knowledge on the "ancestor" of the HERV-K(HML2) family, we asked whether an "average human genome" would still be able to generate fully infectious retroviral particles. With the proviruses that were used to assemble the consensus sequence, we constructed a set of chimeric elements that could spontaneously emerge via recombination between definite human alleles and were able to express all of the retroviral genes. As described in the Methods, the $5^{\prime}$ part was "borrowed" from the HERV-K109 element, the 3' end from K108, K113, or K115 (Fig. 7), and the heterologous CMV promoter was introduced, as for Phoenix, in place of the U3 part of the $5^{\prime}$ LTR. We assayed these three proviruses for a virionassociated RT activity in the supernatant of human 293T cells following their transient transfection and, remarkably, found that the K109/K115 chimera was positive (Figs. 3C, 7). However, this construct is not infectious on its own, most probably because of defects in the HERV-K115 env gene (which is noninfectious in
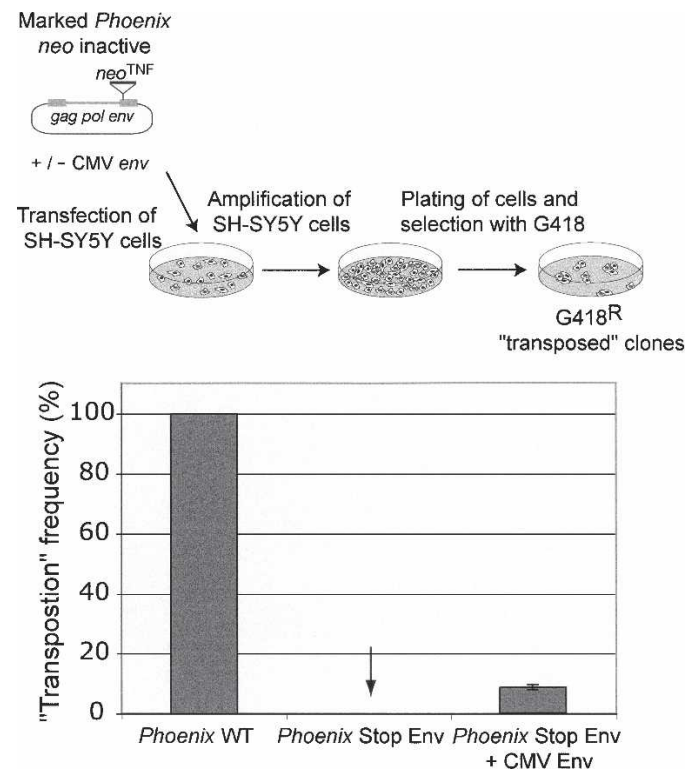

Figure 6. Assay for Phoenix "retrotransposition." (Top) Rationale of the assay. Phoenix was marked with the $n e o^{T N F}$ indicator gene for retrotransposition (Esnault et al. 2002), in which neo becomes active only after a complete retrotransposition cycle (i.e., transcription, reverse transcription, and integration), and the marked element was introduced by transfection into SH-SY5Y human cells permissive for Phoenix infection. The cells were then amplified and subjected to G418 selection for 2 wk; the number of $\mathrm{G} 418^{\mathrm{R}}$ clones (several of which were assayed by PCR to demonstrate splicing out of the intron within the neo indicator gene) yielded the frequency of retrotransposition of the marked elements. (Bottom) "Retrotransposition" is dependent on the presence of a functional env gene, either present within the native Phoenix provirus (Phoenix WT) or added in trans to an env-mutated Phoenix provirus (Phoenix Stop Env) by cotransfection of the cells with an env expression vector (CMV Env). The results presented here correspond to three independent experiments, performed with 5-10 $\times 10^{6}$ cells, and are given as relative transposition frequencies as compared to neo ${ }^{\text {TNF}}$-marked Phoenix (whose transposition frequency is $2 \times 10^{-5}$ clone/seeded cell under these conditions).

\section{Genome Research}

www.genome.org 
RT assay Infection Assay (arbitrary unit)

\begin{tabular}{|c|c|c|c|}
\hline K108 & 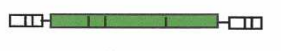 & - & $<2$ \\
\hline K109 & 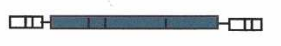 & - & $<2$ \\
\hline K113 & 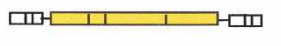 & - & $<2$ \\
\hline K115 & 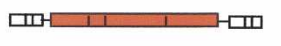 & - & $<2$ \\
\hline K109 / K108 & س & - & $<2$ \\
\hline K109 / K113 & 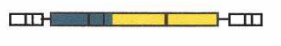 & - & $<2$ \\
\hline K109 / K115 & 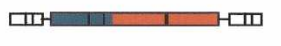 & + & $<2$ \\
\hline K109 / K115 / K108 & 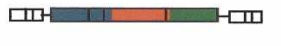 & + & $13 \pm 1.7$ \\
\hline Phoenix & 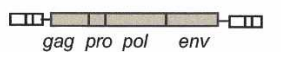 & + & 100 \\
\hline
\end{tabular}

Figure 7. An infectious HERV-K(HML2) retrovirus can be generated by in vitro recombination from cloned $H E R V-K$ loci. Structure of the cloned HERV-K(HML2) proviruses and of the in vitro constructed chimera (see Methods), with the RT activities in the supernatant of 293T cells transfected with the corresponding plasmids measured as in Figure $3 \mathrm{C}$, and the associated infection efficiencies measured by quantitative PCR on the target cell genomic DNA (BHK21 cells, see Methods).

pseudotype experiments, at variance with the K108 env gene) (Dewannieux et al. 2005). Therefore we generated a "triple chimera" by replacing the env region in the above construct with that from K108 and assayed it for infection. With this element, specific integration of the provirus (i.e., not observed with Phoenix mutated in the RT domain used as a control) could be detected in the BHK21 cells by quantitative PCR (see Methods) at a level only five- to 10-fold lower than that observed with Phoenix (Fig. 7). This indicates that, as observed in AKR mice where a multistep recombination process reproductively generates new infectious retroviruses (Stoye et al. 1991; Boeke and Stoye 1997), some individuals could reconstitute functional HERV-K(HML2) proviruses either via a three-fragment recombination event, or more likely via a single recombination event between 2 HERV-K copies (e.g., K109 and K115) with complementation in trans by an intact envelope protein (e.g., K108). Recombination events have indeed occurred frequently between HERV-K(HML2) proviruses (Hughes and Coffin 2005). In this way, or even using functional (but still uncharacterized) alleles that might be present in some individuals, the HERV-K(HML2) family could repeat its life cycle and regenerate functional elements that could, in turn, be the initiators of amplification events to come.

\section{Discussion}

In the present investigation, the reconstruction of an active HERV-K(HML2) provirus gives access de facto to the first identified human retrovirus of endogenous origin that can consequently be characterized at both the structural and functional level. Actually, following "endogenization," which transformed its retroviral status into that of a host genomic locus and thus dramatically reduced its evolutionary rate, this element has been frozen in time in its ancestral state, which we have recovered here. The essential attributes of Phoenix are those of a type-C retrovirus, with assembly and budding at the cell membrane, release in the cell supernatant of particles disclosing protruding spikes that can be unambiguously identified as envelope proteins, morphological maturation of the core structure upon viral protease processing, and finally, infectivity of the particles for a range of target cells. Furthermore, analysis of the integrated proviruses associated with infection discloses the specific structural features of retroviral integration, with precise opening of the target sites, insertion of complete proviral structures with reconstituted LTR, and target-site duplications of a few base pairs. Of note, the target-site duplications are not of a fixed length, but vary from 5 to $6 \mathrm{bp}$. Although this is not common for present-day infectious retroviruses, where the target site duplications are of fixed lengths (for review, see Brown 1997), this peculiarity is also observed for the endogenous proviral $H E R V-K$ loci, as illustrated in Figure 5. Possibly, this unusual feature might reflect the "age" of Phoenix and be a specific characteristic of its ancestral integrase.

Another particular trait of Phoenix is its low infectious titer. One possible interpretation could be that this results from its sensitivity to one or more of the multiple cellular defense mechanisms that have evolved to counteract the amplification of retroelements, thus guarding their hosts against the potentially harmful consequences of retrotransposition. The replication of Phoenix could be limited by RNA interference-mediated gene silencing, as well as by the action of a variety of host gene-encoded restriction factors, such as members of the AID/APOBEC cytosine deaminase family or of the TRIM protein family (for review, see Goff 2004; Harris and Liddament 2004). Another possible interpretation could be that the still not-identified receptor for this ancestral retrovirus has diverged since its entry into the primate genome and is no more adapted to Phoenix, and could even have been positively selected for this divergence for the sake of the host. However, a more likely interpretation, consistent with the frequently observed images shown in Figure $4 \mathrm{~B}$, is that its mode of transmission is more closely related to that of retroviruses such as human HTLV, where virions are poorly infectious and spread essentially via cell-cell contacts, most probably at the level of dedicated "virological synapses" (for review, see Jolly and Sattentau 2004). Similar structures might exist for Phoenix, also consistent with its relative phylogenetic proximity to delta- (HTLV) and lenti- (HIV) viruses (Tristem 2000; Benit et al. 2001), and should actually be particularly well suited to optimize cell reinfection and proviral amplification. As a matter of fact, despite its low infectious titer, another important and related outcome of the present investigation is the demonstration that past amplification of $H E R V-K$ has most probably involved an extracellular pathway and cell reinfection: Indeed, using an appropriate retrotransposition assay, we demonstrate that amplification requires the presence of a functional env gene, and is only observed with cells that are permissive for infection. Accordingly, the mode of amplification of Phoenix is clearly distinct from that prevailing for most retroelements naturally present in eukaryote genomes, which amplify via a strictly intracellular pathway. These elements include the highly reiterated LINE and SINE sequences, which are non-LTR retrotransposons that replicate also by a copy-and-paste mechanism but are not infectious, and the classical LTR retrotransposons, such as the yeast Ty1 and the Drosophila copia elements, which are closely related to retroviruses, of which they are most probably the evolutionary precursors but do not possess an env gene and transpose intracellularly (for review, see Boeke and Stoye 1997; Kazazian Jr. 2004; Dewannieux and Heidmann 2005). In this respect, the HERV-K family of elements has a distinct and specific mode of amplification, which very likely also applies to most of the so-called "endogenous retroviruses," and Phoenix, being actually the first infectious 
HERV available to date, can therefore be considered as a model element to characterize the life cycle of these invaders of all mammalian genomes.

Finally, the present active cloned element provides a potent tool to appraise the role of elements of the HERV-K family in a range of diseases where related particles and viral proteins have been detected. These include essentially, but not exclusively, human tumors such as germ-line tumors and melanomas, where such elements have been suggested to play a role (Boller et al. 1993; Löwer et al. 1993, 1996; Bieda et al. 2001; Muster et al. 2003; Buscher et al. 2005) (for review, see Bannert and Kurth 2004). Taking into account their retroviral origin, firmly established by the present investigation, it is indeed legitimate to hypothesize that HERV-K elements have conserved some of the pathological potency of infectious retroviruses, which could arise upon their transcriptional activation. Clearly, most present-day infectious retroviruses are pathogenic and mediate such effects via both insertional mutagenesis and the activity of the viral proteins they encode. The present study clearly shows that Phoenix is a complex retrovirus that can insert into new genomic locations, and that most probably, human cells can generate active retroviruses by recombination between genomic loci. As shown in the Results section, in vitro recombination between cloned HERV-K copies indeed generate a functional element (and active when further placed under the control of the strong CMV promoter) and it is likely, taking into account the polymorphism of such elements in the human population, that some alleles might still be functional and even become fully active in some definite circumstances. Search for such alleles, possibly among individuals with specific tumors where HERV-K elements are expressed and viral particles detected, should now be performed using Phoenix as a functional "reference" element together with the presently devised infectivity assay.

Altogether, the present results have allowed the structural and functional characterization of the oldest active retrovirus isolated to date and the unraveling of the molecular scenario responsible for its successful amplification in primate genomes. They also provide tools for elucidating the role of its "genomic offspring" in human diseases, in particular in some tumors that have primarily been qualified as of genetic origin, but could ultimately turn out to have a bona-fide retroviral basis. In this respect, this study also suggests that more appropriate safety conditions should be considered for the manipulation of such tissues and tumor cell lines.

\section{Methods}

In silico engineering and in vitro reconstruction of the HERV-K(HML2) consensus provirus

To generate the consensus HERV-K(HML2) provirus sequence, we took the nucleotide sequences of the nine full-length, humanspecific, 9.4-kb class proviruses that can be found in sequence databases and publications and aligned them using ClustalW to generate the consensus sequence (see Supplemental Fig. 1; Supplemental Table 1). To obtain a backbone for the Phoenix provirus expression vector, we subcloned the K108 and K109 proviruses by digesting commercial BAC clones RP11-33P21 and RP11-152J15 (BACPAC Resources), and generated chimeric constructs to get closer to the consensus sequence. Fragments from these chimeric constructs were placed in pALTER-1 plasmid (Promega) and subjected to successive rounds of site-directed mutagenesis using the Altered Sites II in vitro Mutagenesis System
(Promega) and appropriate primers. When all expected mutations had been introduced, the Phoenix provirus was reassembled in the pBluescript plasmid and we checked the whole construct by sequencing. To generate the CMV expression vector, the CMV promoter region was isolated from pCMVß (Clontech, EcoRISacI fragment) and used it to replace the U3 part of the 5' LTR, using PCR, so as to keep the transcription start site unchanged.

Point mutations within the protease and RT motifs (from LVDTGA to LVaaaA and from YIDD to aIaa, respectively) were introduced using the Altered Sites II in vitro Mutagenesis System (Promega) and appropriate primers. Inactivation of the gag gene was achieved via an in-phase deletion between the SfoI and PshAI unique sites, and the env gene was disrupted by inserting a small synthetic linker containing Stop codons in all six frames at the unique StuI site (located at the end of the SU subunit, position 7784 in the sequence provided in Supplemental Fig. 1).

This series of expression vectors was marked with the $S V$ neo resistance gene or $S V$ neo ${ }^{T N F}$ retrotransposition indicator gene by inserting fragments taken from pSVneo* and pSVneo ${ }^{\mathrm{TNF}}$ plasmids (Esnault et al. 2002), respectively, at position 8662 of the consensus sequence (position according to the sequence provided in Supplemental Fig. 1).

\section{Construction of chimeric HERV-K proviruses}

To generate the chimeric HERV-K proviruses, we first constructed an expression vector for the HERV-K109 copy by replacing the U3 part of its 5' LTR by the CMV promoter region. The 3' part of this provirus was replaced by the corresponding sequences of K113 and K115 proviruses (from the unique SexAI site to the end) or K108-1 (from the SacI site to the end). For the triple chimera K109-K115-K108, the 3' domain of K109-K115 was replaced by the corresponding part of the K108-1 provirus (from the XbaI site to the end).

\section{Production of antibodies: Western blot analysis and immunofluorescence studies}

We developed a polyclonal anti-HERV-K Gag antiserum by immunizing rabbits with a mix of purified recombinant proteins produced in bacteria, covering the complete HERV-K113 Gag protein. The resulting sera were checked by Immunoblot analyses and affinity-purified before use (Agrobio). The monoclonal antibody directed against the SU subunit of the HERV-K envelope protein has already been described (Dewannieux et al. 2005). We also raised a rabbit antiserum specific for the HERV-K envelope protein using a recombinant protein produced in bacteria, corresponding to aa 223-437 of the K109 Env protein.

For analysis of retroviral protein expression and cleavage, $293 \mathrm{~T}$ cells transiently transfected with the provirus expression vectors (using Lipofectamine and +Reagent; Invitrogen) were lysed in Laemmli buffer $48 \mathrm{~h}$ post-transfection and lysate proteins directly separated by SDS-PAGE, followed by transfer onto nitrocellulose membranes (Schleicher and Schuell) and incubation with the appropriate antibody and with a secondary antibody linked to Horseradish peroxidase. For analysis of cell supernatants, 293T cells were transfected by the Calcium Phosphate method using the MBS commercial kit (Stratagene). Particulate material in the medium was concentrated by ultracentrifugation on a $20 \%$ sucrose cushion $48 \mathrm{~h}$ post-transfection, and the virions were lysed in Laemmli buffer before viral protein analysis by SDS-PAGE and immunoblotting.

Immunofluorescence analysis of the intracellular localization of Gag and Env was carried out on 293T cells transiently transfected with the Phoenix expression vector. Cells were fixed $30 \mathrm{~h}$ post-transfection, permeabilized, and stained with a rabbit

\section{Genome Research}

www.genome.org 
polyclonal anti-Gag serum and a mouse monoclonal anti-Env antibody. Alexa 546-conjugated goat anti-rabbit IgG and Alexa 488-conjugated goat anti-mouse IgG were used as secondary antibodies (Molecular Probes); nuclei were stained with Dapi and Topro3 (Molecular Probes).

\section{Electron microscopy}

For morphological studies, cells were fixed in phosphate buffer ( $\mathrm{pH} 7.2), 3 \%$ glutaraldehyde for $1 \mathrm{~h}$, and post-fixed in $0.1 \mathrm{M}$ cacodylate buffer, 1\% osmium tetroxyde for $2 \mathrm{~h}$. After being rinsed for $5 \mathrm{~min}$ in water and $15 \mathrm{~min}$ in $0.1 \mathrm{M}$ cacodylate buffer, cells were transferred to $0.2 \mathrm{M}$ cacodylate buffer for $30 \mathrm{~min}$. Cells were washed in $30 \%$ methanol for $10 \mathrm{~min}$, stained in $2 \%$ uranyl acetate in $0.1 \mathrm{M}$ cacodylate buffer , 30\% methanol for $1 \mathrm{~h}$, and washed in 30\% methanol. Cells were then dehydrated through a graded ethanol-propylene oxide series and embedded in Epon 812. Ultrathin sections were collected on formvar-carbon-coated gold grids (200-mesh), stained with $4 \%$ uranyl acetate and lead citrate, and examined with a Zeiss 902 microscope at $80 \mathrm{Kv}$.

For immuno-electron microscopy experiments, transfected cells were fixed for $1 \mathrm{~h}$ at $4^{\circ} \mathrm{C}$ with either $4 \%$ formaldehyde or $1.6 \%$ glutaraldehyde in $0.1 \mathrm{M}$ phosphate buffer ( $\mathrm{pH}$ 7.3). During fixation, the cells were scraped from the plates and centrifuged. Pellets were dehydrated in increasing concentrations of methanol and embedded in Lowicryl K4M at low temperature. Polymerization was carried out for $5 \mathrm{~d}$ at $-30^{\circ} \mathrm{C}$ under longwavelength UV light. Ultrathin sections of Lowicryl-embedded material were collected on formvar-carbon-coated gold grids (200-mesh) and used for labeling with a rabbit antiserum specific for the SU subunit of the HERV-K envelope protein, followed by incubation with a goat anti-rabbit IgG conjugated to gold particles, $10 \mathrm{~nm}$ in diameter. Sections were contrasted as above. The specificity of the association between the gold beads and the viral particles was performed by counting, in 11 independent fields, the number of gold particles $/ \mu \mathrm{m}^{2}$ within three "compartments" (viral particles, cytoplasm, and particle-free extracellular space).

\section{RT assay}

Assays for the presence of RT activity in cell supernatants were performed using transiently transfected 293T cells. At day 2 posttransfection, the medium was harvested, filtered through 0.45 $\mu \mathrm{m}$ pore-sized membranes, and particulate material concentrated by ultracentrifugation on a $20 \%$ sucrose cushion. The virions were lysed in Buffer A (25 mM Tris, $0.25 \mathrm{mM}$ EDTA, $50 \mathrm{mM} \mathrm{KCl}$, $5 \mathrm{mM}$ DTT, $0.025 \%$ Triton X-100, 50\% glycerol) and used as a reverse-transcriptase source for reverse transcription of commercial MS2 RNA (adapted from the PERT assay described in Pyra et al. 1994). Briefly, $300 \mathrm{ng}$ of MS2 RNA were annealed to $16 \mathrm{pmol}$ of RT-1 primer and incubated with viral lysate corresponding to 1-2 mL of cell supernatant. cDNA synthesis was then assayed using $1 / 25$ of the RT reaction as a template for a 25 cycle PCR reaction conducted with RT-1 and RT-2 primers (Pyra et al. 1994). In these assays, expression vectors for the MLV and HIV core proteins were used as positive controls and CMV- $\beta$ (Clontech) as a negative control.

\section{Infection and retrotransposition assays}

For infection assays, 293T cells were used as producer cells, following transient transfection with the consensus provirus by the Calcium Phosphate method (Stratagene MBS commercial kit). Target cell lines (SH-SY5Y, BHK21, G355.5, HeLa, and WOP) were seeded the day before infection in 6-well plates. The day of infection, the supernatants of 293T cells were harvested, filtered through $0.45-\mu \mathrm{m}$ pore-sized membranes, and added to target cells ( $1 \mathrm{~mL}$ per well, with $4 \mu \mathrm{g} / \mathrm{mL}$ polybrene). To increase the sensitivity of the assay, the target cells were then subjected to "spinoculation" (centrifugation at $1200 \mathrm{~g}$ for $2.5 \mathrm{~h}$ ) and incubated overnight at $37^{\circ} \mathrm{C}$ before medium change. For detection of infection events, target cells were split into 10 -cm plates $\left(\sim 5 \times 10^{5}\right.$ cells per plate) at day 3 post infection and subjected to G418 selection $24 \mathrm{~h}$ later. For infection assays using quantitative PCR, $10^{5}$ BHK21 cells were seeded in 6-well plates and used as target cells. At day 2 post-infection, the cells were washed with DNaseI to remove residual DNA and split, and genomic DNA was recovered at days 5-6 post-infection. DNA purification was performed via two successive phenol/chloroform extractions followed by ethanol precipitation. Integration events were detected with the Taqman technology using a 5'-FAM MGB probe (6FAMATAGTGTATGGGTACCTGGC-MGBNFQ, Applied Biosystems) and primers specific for the HERV-K(HML2) family (5'-GCCTTT CCCGCCCTTAATT-3' and 5'-GCAGGGCAGCGATCATCTA-3'), and $500 \mathrm{ng}$ of genomic DNA as a template. The infectious titers were estimated by comparison with a standard obtained by mixing genomic DNAs extracted from naive BHK21 cells and from a clone containing a single inserted provirus in varying proportions. In this assay, the titer of Phoenix WT was in the $10^{3}$ infectious particles/milliliter range. BHK21 cells were chosen as the target cell line, since their genomic DNA generates only very limited background with the $H E R V$-K-specific primers and probe used in this assay; genomic DNA of BHK21 cells infected with Phoenix particles deficient for the RT was used as a negative control to rule out contamination by residual DNA that could originate from the transfected plasmid or genomic DNA of the producer cells.

For retrotransposition assays, $7.5 \times 10^{5} \mathrm{SH}-\mathrm{SY} 5 \mathrm{Y}$ cells were seeded in $60-\mathrm{mm}$ dishes the day prior to transfection. Transfection was performed using $8 \mu \mathrm{L}$ of Plus Reagent (Invitrogen), $12 \mu \mathrm{L}$ of Lipofectamine (Invitrogen), and $3 \mu \mathrm{g}$ of DNA (2.5 $\mu \mathrm{g}$ of Phoenix-derived expression vector and $0.5 \mu \mathrm{g}$ of $e n v$ expression plasmid or control plasmid). Cells were grown for $1 \mathrm{wk}$ before plating on $10-\mathrm{cm}$ plates and subsequent selection with G418. The results presented here correspond to three independent experiments performed with 5-10 $\times 10^{6}$ cells and are given as relative transposition frequencies as compared with $n e 0^{\mathrm{TNF}}$-marked Phoenix (whose absolute transposition frequency is $2 \times 10^{-5}$ clone/ seeded cell under these conditions).

\section{Characterization of HERV-K(HML2) de novo insertion sites}

Integration sites were analyzed by inverse PCR using individual G418 ${ }^{\mathrm{R}}$ clones essentially as described (Dewannieux et al. 2003). Briefly, $5 \mu \mathrm{g}$ of DNA from each clone was digested with PvuII and either (1) DraI, (2) SspI, or (3) with a mix of XbaI, AvrII, and SpeI, or (4) with ApoI alone. After enzyme removal, DNA was diluted and self-ligated overnight. It was ethanol-precipitated, dissolved in $20 \mu \mathrm{L}$ of Tris $10 \mathrm{mM}$, and subjected to one (or in some cases two) round of PCR with sets of divergent primers located within the neo gene and designed for the amplification of the $5^{\prime}$ or $3^{\prime}$ end and flanking sequences of the newly inserted proviruses. The amplified DNAs were gel-purified, cloned, and sequenced, thus yielding the target-site sequences. The identification of these insertion sites was further confirmed by PCR reactions allowing direct amplification of both left and right junctions between the newly inserted proviruses and the identified loci, using for each reaction a primer specific for the identified insertion locus and an internal primer located in the neo gene. In each case, we checked that the same reactions performed on the naive, noninfected target cells did not yield any specific amplification. Primer sequences are available on request. 


\section{Safety precautions}

All manipulations involving the reconstructed HERV-K were carried out in our lab according to the rules established by the "Commission de Génie Génétique" from the "Ministère délégué à l'Enseignement supérieur et à la Recherche" French authority that regulates handling of genetically modified organisms in all research institutions in France.

Albeit the HERV-K virus has a very low infectivity and does not sustain multiple-cycle infection, at least in all the cells tested, Phoenix is a retrovirus, and as such, is a priori eligible to BL3 conditions for manipulation. Accordingly, the material will only be sent to other labs in appropriate sealed containers in the form of small amounts of plasmid DNA that will require it to be amplified before use as a transfection vector to produce viral particles. At the present time and as a precautionary principle, it will only be distributed under a material transfer agreement specifying the commitment of the recipient labs to carry out every experiment using the material under BL3 conditions and accompanied by a duly signed authorization form from the Biosafety Committee responsible for genetic manipulations in their country of origin.

\section{Acknowledgments}

We thank A. Jalil for assistance with confocal microscopy, E. Pichard for technical assistance, and C. Lavialle for critical reading of the manuscript. This work was supported by the CNRS and by grants from the Ligue Nationale contre Le Cancer (Equipe Labellisée).

\section{References}

Bannert, N. and Kurth, R. 2004. Retroelements and the human genome: New perspectives on an old relation. Proc. Natl. Acad. Sci. 101: $14572-14579$.

Barbulescu, M., Turner, G., Seaman, M.I., Deinard, A.S., Kidd, K.K., and Lenz, J. 1999. Many human endogenous retrovirus K (HERV-K) proviruses are unique to humans. Curr. Biol. 9: 861-868.

Belshaw, R., Pereira, V., Katzourakis, A., Talbot, G., Paces, J., Burt, A., and Tristem, M. 2004. Long-term reinfection of the human genome by endogenous retroviruses. Proc. Natl. Acad. Sci. 25: 25.

Belshaw, R., Dawson, A.L., Woolven-Allen, J., Redding, J., Burt, A., and Tristem, M. 2005. Genomewide screening reveals high levels of insertional polymorphism in the human endogenous retrovirus family HERV-K(HML2): Implications for present-day activity. J. Virol. 79: $12507-12514$.

Benit, L., Dessen, P., and Heidmann, T. 2001. Identification, phylogeny, and evolution of retroviral elements based on their envelope genes. J. Virol. 75: 11709-11719.

Bieda, K., Hoffmann, A., and Boller, K. 2001. Phenotypic heterogeneity of human endogenous retrovirus particles produced by teratocarcinoma cell lines. J. Gen. Virol. 82: 591-596.

Boeke, J.D. and Stoye, J.P. 1997. Retrotransposons, endogenous retroviruses, and the evolution of retroelements. In Retroviruses (eds. J.M. Coffin et al.), pp. 343-435. Cold Spring Harbor Laboratory Press, Cold Spring Harbor, NY.

Boller, K., König, H., Sauter, M., Mueller-Lantzsch, N., Löwer, R., Löwer, J., and Kurth, R. 1993. Evidence that HERV-K is the endogenous retrovirus sequence that codes for the human teratocarcinoma-derived retrovirus HTDV. Virology 196: 349-353.

Brown, P.O. 1997. Integration. In Retroviruses (eds. J.M. Coffin et al.), pp. 161-204. Cold Spring Harbor Laboratory Press, Cold Spring Harbor, NY.

Buscher, K., Trefzer, U., Hofmann, M., Sterry, W., Kurth, R., and Denner, J. 2005. Expression of human endogenous retrovirus $\mathrm{K}$ in melanomas and melanoma cell lines. Cancer Res. 65: 4172-4180.

Bushman, F.D. 2003. Targeting survival. Integration site selection by retroviruses and LTR-retrotransposons. Cell 115: 135-138.

de Parseval, N., Lazar, V., Bénit, L., Casella, J., and Heidmann, T. 2003. Survey of human genes of retroviral origin: Identification and transcriptome of the genes with coding capacity for complete envelope proteins. J. Virol. 77: 10414-10422.
Dewannieux, M. and Heidmann, T. 2005. LINEs, SINEs and processed pseudogenes: Parasitic strategies for genome modeling. Cytogenet. Genome Res. 110: 35-48.

Dewannieux, M., Esnault, C., and Heidmann, T. 2003. LINE-mediated retrotransposition of marked Alu sequences. Nat. Genet. 35: 41-48.

Dewannieux, M., Blaise, S., and Heidmann, T. 2005. Identification of a functional envelope protein from the HERV-K family of human endogenous retroviruses. J. Virol. 79: 15573-15577.

Dirksen, E. and Levy, J. 1977. Virus-like particles in placentas from normal individuals and patients with systemic lupus erythematosus. J. Natl. Cancer Inst. 59: 1187-1192.

Esnault, C., Casella, J.F., and Heidmann, T. 2002. A Tetrahymena thermophila ribozyme-based indicator gene to detect transposition of marked retroelements in mammalian cells. Nucleic Acids Res. 30: e49.

Goff, S. 2004. Retrovirus restriction factors. Mol. Cell 16: 849-859.

Harris, R.S. and Liddament, M.T. 2004. Retroviral restriction by APOBEC proteins. Nat. Rev. Immunol. 4: 868-877.

Hughes, J.F. and Coffin, J.M. 2004. Human endogenous retrovirus K solo-LTR formation and insertional polymorphisms: Implications for human and viral evolution. Proc. Natl. Acad. Sci. 101: 1668-1672.

Hughes, J.F. and Coffin, J.M. 2005. Human endogenous retroviral elements as indicators of ectopic recombination events in the primate genome. Genetics 12: 12 .

Jolly, C. and Sattentau, Q.J. 2004. Retroviral spread by induction of virological synapses. Traffic 5: 643-650.

Kalter, S.S., Helmke, R.J., Heberling, R.L., Panigel, M., Fowler, A.K., Strickland, J.E., and Hellman, A. 1973. C-Type particles in normal human placentas. J. Natl. Cancer Inst. 50: 1081-1084.

Kazazian Jr., H.H. 2004. Mobile elements: Drivers of genome evolution. Science 303: 1626-1632.

Lavie, L., Kitova, M., Maldener, E., Meese, E., and Mayer, J. 2005. CpG methylation directly regulates transcriptional activity of the human endogenous retrovirus family HERV-K(HML-2). J. Virol. 79: 876-883.

Löwer, R., Boller, K., Hasenmaier, B., Korbmacher, C., Mueller-Lantzsch, N., Löwer, J., and Kurth, R. 1993. Identification of human endogenous retroviruses with complex mRNA expression and particle formation. Proc. Natl. Acad. Sci. 90: 4480-4484.

Löwer, R., Löwer, J., and Kurth, R. 1996. The viruses in all of us: Characteristics and biological significance of human endogenous retrovirus sequences. Proc. Natl. Acad. Sci. 93: 5177-5184.

Mayer, J., Sauter, M., Racz, A., Scherer, D., Mueller-Lantzsch, N., and Meese, E. 1999. An almost-intact human endogenous retrovirus K on human chromosome 7. Nat. Genet. 21: 257-258.

Medstrand, P. and Mager, D.L. 1998. Human-specific integrations of the HERV-K endogenous retrovirus family. J. Virol. 72: 9782-9787.

Muster, T., Waltenberger, A., Grassauer, A., Hirschl, S., Caucig, P., Romirer, I., Fodinger, D., Seppele, H., Schanab, O., Magin-Lachmann, C., et al. 2003. An endogenous retrovirus derived from human melanoma cells. Cancer Res. 63: 8735-8741.

Pyra, H., Boni, J., and Schupbach, J. 1994. Ultrasensitive retrovirus detection by a reverse transcriptase assay based on product enhancement. Proc. Natl. Acad. Sci. 91: 1544-1548.

Reus, K., Mayer, J., Sauter, M., Scherer, D., Muller-Lantzsch, N., and Meese, E. 2001. Genomic organization of the human endogenous retrovirus HERV-K(HML-2.HOM) (ERVK6) on chromosome 7. Genomics 72: 314-320.

Ruda, V.M., Akopov, S.B., Trubetskoy, D.O., Manuylov, N.L., Vetchinova, A.S., Zavalova, L.L., Nikolaev, L.G., and Sverdlov, E.D. 2004. Tissue specificity of enhancer and promoter activities of a HERV-K(HML-2) LTR. Virus Res. 104: 11-16.

Steinhuber, S., Brack, M., Hunsmann, G., Schwelberger, H., Dierich, M.P., and Vogetseder, W. 1995. Distribution of human endogenous retrovirus HERV-K genomes in humans and different primates. Hum. Genet. 96: 188-192.

Stoye, J.P., Moroni, C., and Coffin, J.M. 1991. Virological events leading to spontaneous AKR thymomas. J. Virol. 65: 1273-1285.

Tristem, M. 2000. Identification and characterization of novel human endogenous retrovirus families by phylogenetic screening of the human genome mapping project database. J. Virol. 74: 3715-3730.

Turner, G., Barbulescu, M., Su, M., Jensen-Seaman, M.I., Kidd, K.K., and Lenz, J. 2001. Insertional polymorphisms of full-length endogenous retroviruses in humans. Curr. Biol. 11: 1531-1535.

Villesen, P., Aagaard, L., Wiuf, C., and Pedersen, F.S. 2004. Identification of endogenous retroviral reading frames in the human genome. Retrovirology 1: 32.

Wilkinson, D.A., Mager, D.L., and Leong, J.A.C. 1994. Endogenous human retroviruses. In The retroviridae (ed. J.A. Levy), pp. 465-535. Plenum Press, New York.

Received May 28, 2006; accepted in revised form August 30, 2006.

\section{Genome Research}

www.genome.org 


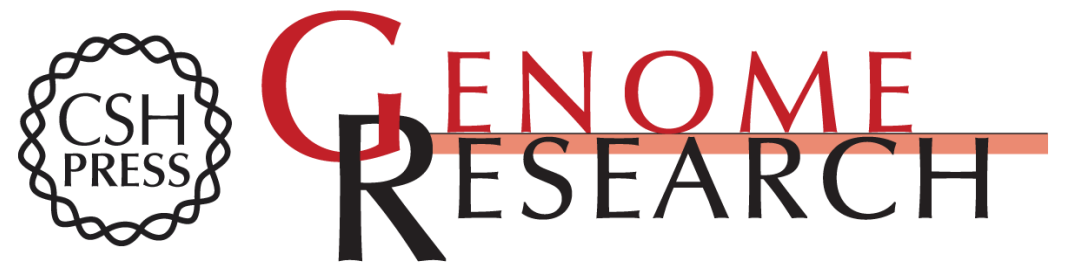

\section{Identification of an infectious progenitor for the multiple-copy HERV-K human endogenous retroelements}

Marie Dewannieux, Francis Harper, Aurélien Richaud, et al.

Genome Res. 2006 16: 1548-1556 originally published online October 31, 2006

Access the most recent version at doi:10.1101/gr.5565706

Supplemental Material

References

License

Email Alerting Service
http://genome.cshlp.org/content/suppl/2006/11/06/gr.5565706.DC1

This article cites 34 articles, 16 of which can be accessed free at: http://genome.cshlp.org/content/16/12/1548.full.html\#ref-list-1

Receive free email alerts when new articles cite this article - sign up in the box at the top right corner of the article or click here.

\section{Affordable, Accurate Sequencing.}

To subscribe to Genome Research go to:

https://genome.cshlp.org/subscriptions 